\title{
Self-induced Structure in the Current-Voltage Characteristics of RSQUIDs
}

\author{
Colin M. Pegrum and John C. Macfarlane
}

\begin{abstract}
Resistive two-junction SQUIDs (RSQUIDs) made from high-temperature superconductors are being developed as narrow-linewidth tunable oscillators in the $\mathbf{G H z}$ frequency range. We present here the results of numerical simulation of RSQUIDs of this type. These studies have identified conditions where sub-harmonic steps and other features are apparent in the current-voltage characteristics, driven by the internally-generated heterodyne frequency. The behavior is sensitive to the frequency (set by the voltage across the resistive element in the RSQUID), the temperature and also the loop inductance. We have studied the effects of thermal noise on these features. We also assess how these effects might be observed, and consider how they might affect practical applications of high frequency heterodyne RSQUID oscillators.
\end{abstract}

Index Terms-Heterodyning, Josephson oscillators, SQUIDs, superconducting devices.

\section{INTRODUCTION}

W HEN a small resistance $R_{\mathrm{S}}$ is added in series with the loop of a SQUID it becomes a resistive SQUID or RSQUID. The first RSQUIDs were RF-biased and were used for noise thermometry (e.g. [1]), calorimetry [2] and thermal measurements [3]. Harding and Zimmerman [4] described a DC-biased 2-junction RSQUID using mechanical niobium point-contact junctions, and Verschueren et al. [5] made a $\mathrm{Pb}-\mathrm{Sn}$ solder-blob version, but only relatively recently have thin-film versions of the DC RSQUID been reported [6]-[12].

\section{MotivaTion}

Most DC RSQUIDs have been used for noise thermometry from $4.2 \mathrm{~K}$ down to the $\mathrm{mK}$ range, but Krivoy and Koch [6] have recently demonstrated a thin-film niobium DC RSQUID as a current-controlled oscillator in the frequency range of a few $\mathrm{kHz}$ to several hundred MHz. This works by feeding an external current $I_{\mathrm{S}}$ through the series resistor $R_{\mathrm{S}}$. The average voltages across the two junctions then differ by $V_{\mathrm{s}}=I_{\mathrm{s}} R_{\mathrm{s}}$ and the different Josephson frequencies mix together by currents coupled through $R_{\mathrm{S}}$ to produce a heterodyne frequency $f_{\mathrm{h}}=\left(I_{\mathrm{s}} R_{\mathrm{s}}\right) / \Phi_{0}$, where $\Phi_{0}$ is the flux quantum.

Hao et al. [13], [14] reported the first HTS current-controlled RSQUID oscillator, operating from 20 to $60 \mathrm{~K}$ at 1 to $50 \mathrm{MHz}$.

Manuscript received August 18, 2008. First published June 30, 2009; current version published July 10, 2009.

C. Pegrum is with FieldSolutions, Glasgow G12 9SD and also with the Department of Physics, University of Strathclyde, Glasgow G4 0NG, UK (e-mail: fieldsolutions@colinpeg2.plus.com).

J. Macfarlane is with the Department of Physics, University of Strathclyde, Glasgow G4 0NG, U.K. (e-mail: j.c.macfarlane@ strath.ac.uk).

Digital Object Identifier 10.1109/TASC.2009.2019591

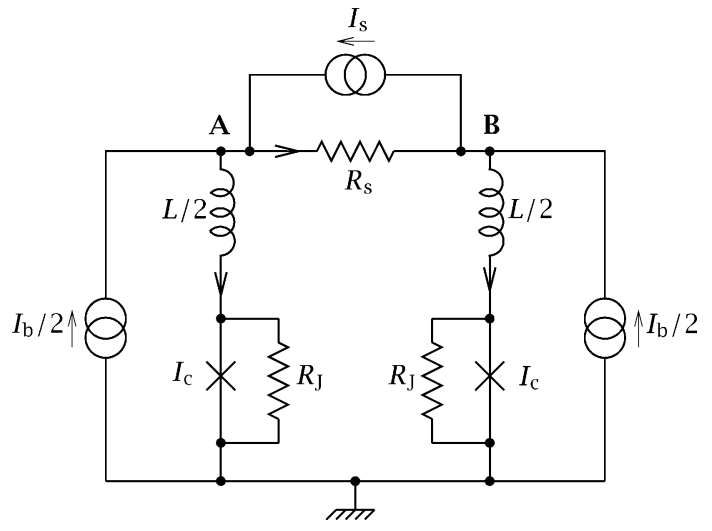

Fig. 1. Circuit model used for the RSQUID. The currents $I_{\mathrm{b}}$ and $I_{\mathrm{s}}$ are supplied by external DC sources. Junction capacitances are zero.

They have recently demonstrated HTS RSQUIDs in the $1 \mathrm{GHz}$ range [15], [16]. To support this recent work we developed models using the JSIM and JSPICE3 circuit analysis software packages, mainly to look at the behavior in the time domain. In this paper we use these models to look at the current-voltage $(I-V)$ curves for DC RSQUIDs.

\section{The RSQUID EQuivalent CIRCUIT AND Model}

Our model has identical shunted Josephson junctions with critical currents $I_{\mathrm{c}}$ and shunt resistors $R_{\mathrm{J}}$, as in Fig. 1 . The junction capacitance is negligible. In the model two equal DC bias currents $I_{\mathrm{b}} / 2$ are fed to each half of the RSQUID. A DC current source feeds the current $I_{\mathrm{s}}$ through the series resistor $R_{\mathrm{s}}$. The total loop inductance $L$ is split into two equal halves.

Practical systems (e.g. [10], [14], [16]) usually minimize external connections and have a different grounding setup and may have just a single bias current, fed to either point $\mathbf{A}$ or $\mathbf{B}$ in Fig. 1, and then $I_{\mathrm{b}}$ will add to $I_{\mathrm{s}}$. For our symmetric model, with three separate current sources, $I_{\mathrm{b}}$ and $I_{\mathrm{S}}$ can be varied independently without interaction. The time-averaged voltage $V_{\text {out }}$ across the RSQUID is measured at point $\mathbf{A}$ or $\mathbf{B}$.

\section{Key QUANTITIES INFLUENCING RSQUID BEHAVIOR}

\section{A. The Heterodyne Frequency $f_{\mathrm{h}}$}

The voltage $V_{\mathrm{s}}=I_{\mathrm{s}} R_{\mathrm{s}}$ across the series resistor $R_{\mathrm{s}}$ can be varied over a very wide range by externally controlling $I_{\mathrm{S}}$, and can alter $f_{\mathrm{h}}$ from the $\mathrm{kHz}$ to $\mathrm{GHz}$ range. Suppose initially that $f_{\mathrm{h}}$ is much less than the Josephson frequency of either junction. Both junctions then have Josephson frequencies of the order of 
$V_{\mathrm{J}} / \Phi_{0}$, where the junction characteristic voltage $V_{\mathrm{J}}=I_{\mathrm{c}} R_{\mathrm{J}}$. We introduce a parameter $\eta$ to compare $f_{\mathrm{h}}$ with $f_{\mathrm{J}}=V_{\mathrm{J}} / \Phi_{0}$ :

$$
\eta=\frac{f_{\mathrm{h}}}{f_{\mathrm{J}}}=\frac{V_{\mathrm{s}}}{V_{\mathrm{J}}}=\frac{I_{\mathrm{s}} R_{\mathrm{s}}}{I_{\mathrm{c}} R_{\mathrm{J}}}
$$

$\eta$ distinguishes between the low and high frequency regimes for $f_{\mathrm{h}}$. In the low-frequency limit $\eta \ll 1$ and $f_{\mathrm{h}}$ is much less than the Josephson frequency of either junction. All RSQUIDs made to date operate in this low-frequency limit. Simulations for one such device are in Section V. (Note that the actual average voltage across the RSQUID under typical operating conditions at fixed $I_{\mathrm{b}}$ is somewhat higher than $V_{\mathrm{J}}$, but only by a factor of $\approx 1.5-2$.)

If $\eta>\approx 0.1$, say, then $f_{\mathrm{h}}$ is a significant fraction of $f_{\mathrm{J}}$, and in this higher-frequency regime the behavior becomes more complicated and new features are apparent. A simulation in this limit is considered in Section VI.

\section{B. The Loop Inductance $L$}

The simulations to follow show that $L$ has a significant effect on both the shape of the $I-V$ and the performance of the RSQUID as a current-controlled oscillator. This issue has been addressed in more detail in [17]. In simple terms the reactance $X_{L}$ added by $L$ at $f_{\mathrm{J}}$ is generally much larger than $R_{\mathrm{s}}$ (and it is often greater than $R_{\mathrm{J}}$ ) and so it can dominate the loop impedance and strongly influence the DC RSQUID dynamics. As $L$ increases, the coupling between the two junctions at their Josephson frequencies around the loop through $R_{\mathrm{s}}$ is progressively reduced. The reactance $X_{L}$ of half the loop inductance at a Josephson frequency $f_{\mathrm{J}}=I_{\mathrm{c}} R_{\mathrm{J}} / \Phi_{0}$ is

$$
X_{L}=2 \pi f_{\mathrm{J}} L / 2=\frac{\pi I_{\mathrm{c}} R_{\mathrm{J}} L}{\Phi_{0}}=\beta_{L} R_{\mathrm{J}},
$$

where $\beta_{L}=\left(\pi I_{\mathrm{c}} L\right) / \Phi_{0}$ and so $\beta_{L}$ is just the ratio $X_{L} / R_{\mathrm{J}}$. For HTS RSQUIDs $R_{\mathrm{J}} \gg R_{\mathrm{S}}$, so the magnitude of $R_{\mathrm{S}}$ has little effect on the coupling.

For an HTS RSQUID it is important to consider its total loop inductance. Its thin-film layout needs a large contact area between the $\mathrm{YBaCuO}$ film and the $\mathrm{Au}$ film forming $R_{\mathrm{S}}$ (to minimize contact resistance) and so there is unavoidably a relatively large distributed stray inductance $L_{\mathrm{s}}$ in series with $R_{\mathrm{s}}$, which adds to the overall loop inductance. It is not straightforward to calculate $L_{\mathrm{s}}$ exactly, but generally it is significantly greater than the inductance of the purely superconducting part of the RSQUID loop.

Note that in our model (Fig. 1) $L$ includes $L_{\mathrm{S}}$, since the steady external current $I_{\mathrm{s}}$ only serves to maintain a difference between the average junction voltages; the effect of $I_{\mathrm{s}}$ flowing through $L_{\mathrm{s}}$ has no effect on the circuit dynamics.

\section{JSIM and JSPICE Analysis}

The work in this paper has mainly used a version of JSIM [18] with added current-noise sources [19] so the effects of temperature $T$ can be studied. JSIM outputs data sampled typically on a picosecond timescale and to generate $I-V$ curves we use a range of additional programs, which apply a low-pass filter to the output with an appropriate cut-off frequency, followed by data averaging, to obtain an average voltage for a set of $I_{\mathrm{b}}$ values. The method has been tested previously against a wide range of junction and SQUID models to confirm its validity [20]. The filters are implemented as $L C R$ second-order filters within the JSIM circuit itself.

Some cross-checks have been done with JSPICE3 [21], but this version of JSPICE does not have noise sources, so it is limited to $T=0$. Results from JSIM and JSPICE3 for circuits that have been compared have been identical.

\section{AN EXAMPLE OF A LOW-FREQUENCY DC RSQUID}

We use parameters similar to those for devices studied experimentally at $T=32 \mathrm{~K}$ [15], [16], with $I_{\mathrm{c}}=217.5 \mu \mathrm{A}$, $R_{\mathrm{J}}=4 \mathrm{ohm}$ and $R_{\mathrm{s}}=8 \times 10^{-3} \mathrm{ohm}$. These have step-edge junctions and we assume their capacitance is negligible. With $I_{\mathrm{S}}$ set to $297 \mu \mathrm{A}, f_{\mathrm{h}} \approx 1.15 \mathrm{GHz}$, as confirmed by measurement. Although $f_{\mathrm{h}}$ is much higher than for the DC RSQUID current-controlled generators reported previously [6], [13], [14], it is still in the low-frequency regime, since $\eta=2.7 \times 10^{-3}$ from (1).

We estimated that the total inductance $L$ was in the range 20-40 $\mathrm{pH}$ and so the device was modeled with different values of $L$ up to $40 \mathrm{pH}$. Fig. 2 shows the results. The uppermost curve (open-circle points) is for $I_{\mathrm{S}}=0$ (it is for $L=20 \mathrm{pH}$, but its shape is independent of $L$.) For $I_{\mathrm{s}}=0$ the device acts like two junctions in parallel with a common Josephson frequency. The degree of noise-rounding is measured by the parameter $\Gamma$ [22] defined for a single junction as

$$
\Gamma=\frac{2 \pi k_{\mathrm{B}} T}{I_{\mathrm{C}} \Phi_{0}}
$$

where $k_{\mathrm{B}}$ is Boltzmann's constant. For the two junctions in parallel at $T=32 \mathrm{~K}, \Gamma=3.2 \times 10^{-3}$, a value that is consistent with the modest degree of rounding in the uppermost $I-V$ curve in Fig. 2. When $I_{\mathrm{S}}$ is turned on in the model, the shape of the $I-V$ curve is altered significantly. The change from the initial noise-rounded $I-V$ curve becomes increasingly marked as $L$ is reduced. All the data points in Fig. 2 are for $T=32 \mathrm{~K}$, but the solid line was modeled independently for $L=2 \mathrm{pH}$ in a separate run for $T=0$.

Experimental tests on an HTS device with the above parameters showed no significant change in the shape of the $I-V$ curve when $I_{\mathrm{s}}$ was turned on or off. This suggests that $L$ was indeed at least $20 \mathrm{pH}$ or more, as was estimated.

Closer examination of the data for the $I-V$ curve at $T=$ 0 for $L=2 \mathrm{pH}$, for example, shows that below about 450 $\mu \mathrm{A}$ it is made up of a set of Shapiro steps of small amplitude, self-induced by the heterodyne signal at $f_{\mathrm{h}}$, and it is the presence of these steps that causes the marked change in shape of the $I-V$ curve, even for $T=32 \mathrm{~K}$. Indeed, the modified shape of the $I-V$ curve for low values of $L$ is similar to what is seen for a single junction strongly coupled to an external low-frequency microwave source.

For this example with $f_{\mathrm{h}}=1.15 \mathrm{GHz}$, the step spacing is approximately $2.4 \mu \mathrm{V}$. Fig. 3 shows an enlarged region of the $I-V$ curve for $L=2 \mathrm{pH}$, for $0<T<1 \mathrm{~K}$. The step height is small, so noise washes them out for $T>\approx 1 \mathrm{~K}$. This is expected: the 


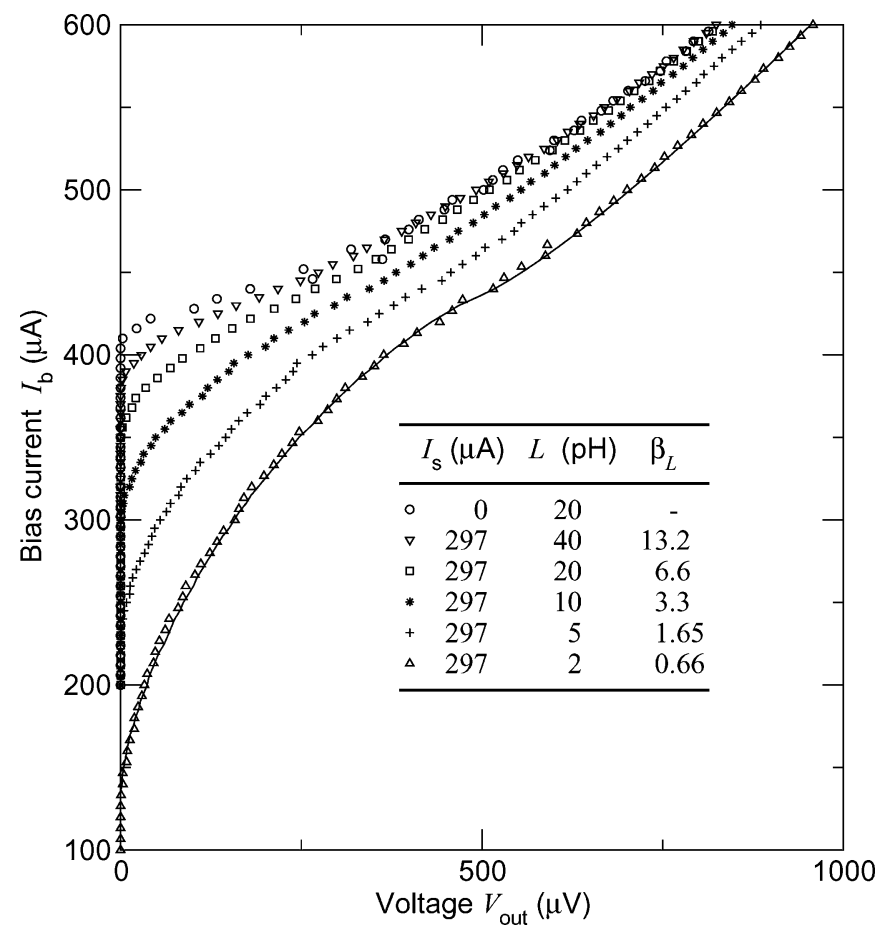

Fig. 2. $I-V$ curves for different values of $L$ for an RSQUID in the lowfrequency limit. All data points are for $T=32 \mathrm{~K}$, but the solid line is for $L=2 \mathrm{pH}$ at $T=0$. The uppermost curve is for no current through $I_{\mathrm{s}}$ and under these conditions the RSQUID behaves like a parallel pair of single noise-rounded resistively-shunted junctions.

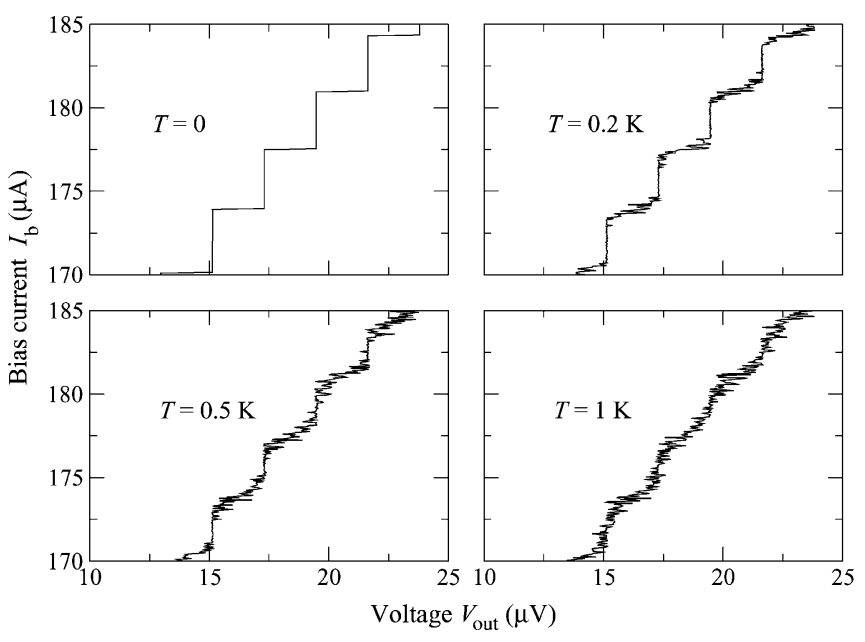

Fig. 3. An enlarged part of the $I-V$ curve of Fig. 2 for $L=2 \mathrm{pH}$ at $0<$ $T<1 \mathrm{~K}$. The bias current increment was $0.025 \mu \mathrm{A}$ for these figures.

noise-rounding parameter $\Gamma_{\mathrm{s}}$ for a microwave-induced step of height $\Delta I_{\mathrm{c}}$ is given by [23]

$$
\Gamma_{\mathrm{s}}=\frac{2 \pi k_{\mathrm{B}} T}{\Delta I_{\mathrm{c}} \Phi_{0}},
$$

which for the steps in Fig. 3 is $\approx 1.2 \times 10^{-2}$ for $T=1 \mathrm{~K}$. Simulations for larger $L$ still show steps at $T=0$, but these have increasingly smaller amplitudes as $L$ is increased, so $T$ would have to be well below $1 \mathrm{~K}$ for these to be seen. So for the device reported in [16], the self-induced steps will have no directlyobservable or adverse effects on its behavior as an oscillator.

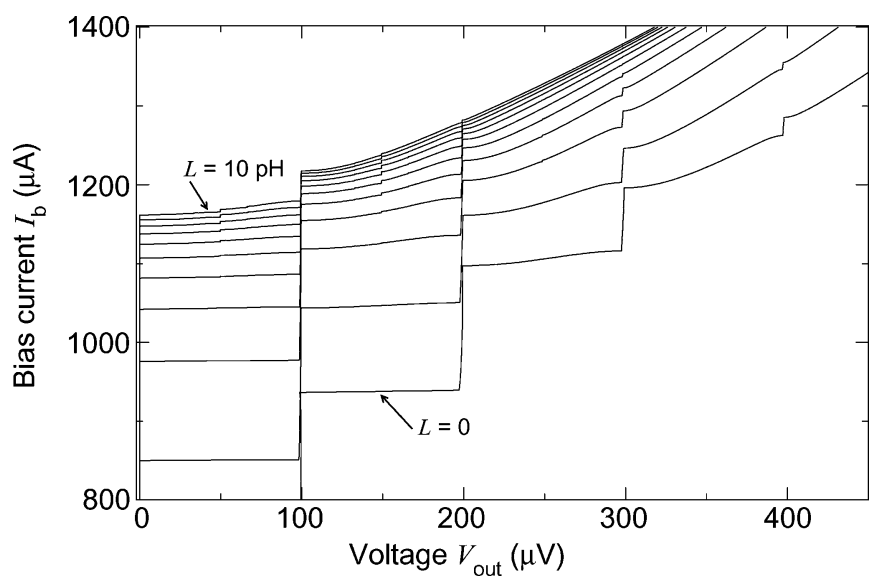

Fig. 4. Simulation at $T=0$, with $I_{\mathrm{c}}=600 \mu \mathrm{A}, R_{\mathrm{s}}=0.02 \mathrm{ohm}$ and $I_{\mathrm{s}}=5 \mathrm{~mA}$. The loop inductance $L$ has been varied in steps of $1 \mathrm{pH}$ from zero (lowermost curve) to $10 \mathrm{pH}$ (top curve). The bias current was incremented in $0.5 \mu \mathrm{A}$ steps for each curve.
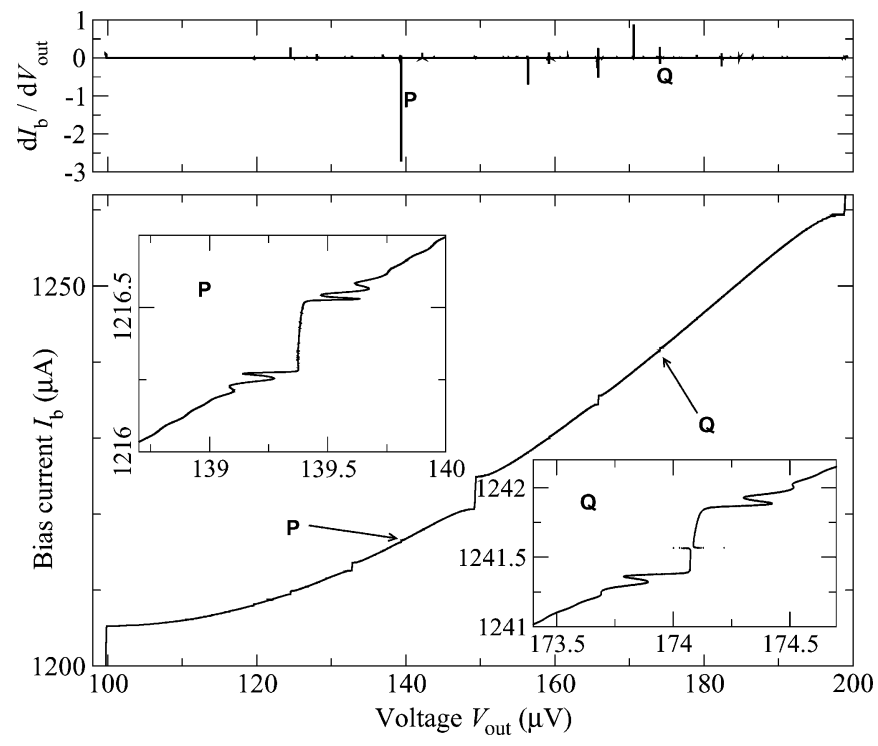

Fig. 5. An expanded view of the $L=7 \mathrm{pH}$ curve for $100<V_{\text {out }}<200 \mu \mathrm{V}$ from Fig. 4, showing sub-harmonic steps. For this main curve $I_{\mathrm{b}}$ increments in steps of $5 \mathrm{nA}$. The top graph shows $d I_{\mathrm{b}} / d V_{\text {out }}$ (on an arbitrary scale); where $d I_{\mathrm{b}} / d V_{\text {out }}$ has a significant negative value, there is more complex fine structure in the $I-V$ curve, as shown in the inset graphs for points $\mathbf{P}$ and $\mathbf{Q} . I_{\mathrm{b}}$ has been stepped in increments of $0.1 \mathrm{nA}$ and $0.2 \mathrm{nA}$ for the expansions around points $\mathbf{P}$ and $\mathbf{Q}$ respectively.

\section{HIGH-FREQUENCY BEHAVIOR}

Here we look at a device at $T=0$ for which $I_{\mathrm{s}} R_{\mathrm{s}}=100 \mu \mathrm{V}$, so $f_{\mathrm{h}} \approx 48 \mathrm{GHz}$. This is purely a simulation study: no device matching these parameters has been made or tested yet. We chose $I_{\mathrm{c}}=600 \mu \mathrm{A}, R_{\mathrm{J}}=1 \mathrm{ohm}, R_{\mathrm{s}}=0.02 \mathrm{ohm}$ and $I_{\mathrm{s}}=5 \mathrm{~mA}$, making $\eta=0.13$. Fig. 4 shows once again that the self-induced step amplitude greatly diminishes as $L$ is increased towards $10 \mathrm{pH}$.

The larger steps show sub-harmonic structure in some regions, notably between the first and second $100 \mu \mathrm{V}$ steps, and its amplitude passes through a maximum for $L \approx 7 \mathrm{pH}$. Fig. 5 shows this in more detail. The differential of the $I-V$ data reveals the locations of sub-harmonic steps and of some other features. Examples are shown in the inset graphs of Fig. 5. These 
"wiggles" have been modeled using very closely-spaced values of $I_{\mathrm{b}}(0.1 \mathrm{nA}$ or $0.2 \mathrm{nA})$ and they are completely deterministic and are not themselves evidence of a region of chaotic behavior. (This is expected, since the junction capacitance is zero in the model we use here and so the differential equations describing the RSQUID are are first-order.) The wiggles have some similarities to the "tails"" seen in RF-driven junctions and SQUIDs [24], [25]

It is important to remember that from (4) any current step or feature must have an amplitude at $1 \mathrm{~K}$ of $\approx 0.4 \mu \mathrm{A}$ or more (for $\Gamma_{\mathrm{s}}=0.1$ ), if it is not to be washed out by thermal noise. So some of the fine structure seen in Fig. 5 might be difficult to see experimentally, other than perhaps the $1 / 3,1 / 2$ and $2 / 3$ sub-harmonic steps, unless ultra-low temperatures are used. However, for $L \approx 3 \mathrm{pH}$ or less, some of the larger integer steps could still be detectable up to about $30 \mathrm{~K}$.

We have also looked at devices with even higher values of $\eta$, attained by progressively reducing $I_{\mathrm{c}}$ from 600 to $100 \mu \mathrm{A}$. Even for $I_{\mathrm{c}}=100 \mu \mathrm{A}$, for which $\eta=1.0$, no additional features of great interest are present in the $I-V$ curves.

\section{CONCLUSIONS}

The $I-V$ curves of DC RSQUIDs clearly can show some interesting features due to Shapiro steps self-induced by the internal heterodyne frequency. The features are most apparent when the total loop inductance $L$ is small, including the contribution $L_{\mathrm{S}}$ from the thin-film structure forming $R_{\mathrm{s}}$. Existing HTS RSQUIDs have a relatively large value of $L_{\mathrm{S}}$ and so for these the self-induced features may not be visible.

In the limit of low heterodyne frequency (typically $1 \mathrm{GHz}$, as recently reported [16]) the step amplitude will be small and at the normal operating temperatures the step structure will be completely masked by thermal noise. The steps will only be discernable for $T<1 \mathrm{~K}$. Nonetheless, for small $L$ the $I-V$ curve at any temperature is still modified in shape by the heterodyne oscillation.

Our simulations show that for a high-frequency device $\left(f_{\mathrm{h}} \approx\right.$ $50 \mathrm{GHz}$ ) large-amplitude integer steps appear, along with subharmonic steps and other features. Some integer steps could be visible at $\approx 30 \mathrm{~K}$ and so these could affect the choice of the operating bias current if the device were used as an oscillator at such a temperature. But the sub-harmonic structure is generally too fine to be seen at HTS operating temperatures.

\section{ACKNOWLEDGMENT}

The authors thank Mesaplexx Pty Ltd, Eight Mile Plains, Brisbane, Australia for their support and interest.

\section{REFERENCES}

[1] R. J. Soulen, W. E. Fogle, and J. H. Colwell, "Measurements of absolute temperature below $0.75 \mathrm{~K}$ using a Josephson-junction noise thermometer," J. Low Temp. Phys., vol. 94, no. 5, pp. 385-487, Mar. 1994.
[2] J. G. Park and A. W. Vaidya, "Resistive SQUID calorimetry at low temperatures," J. Low Temp. Phys., vol. 40, no. 3, pp. 247-274, Aug. 1980.

[3] J. G. Park and A. Vaidya, "Resistive SQUIDs for thermal measurements," IEEE Trans. Magn., vol. 17, no. 1, pp. 845-848, Jan. 1981.

[4] J. T. Harding and J. E. Zimmerman, "Quantum phase fluctuations in resistive circuits containing weakly superconducting junctions," J. Appl. Phys., vol. 41, no. 4, pp. 1581-1588, Mar. 1970.

[5] J. M. V. Verschueren, A. A. Uiterwaal, and R. W. van der Heijden, "Tunable narrow-linewidth oscillations and current-voltage characteristics of a resistive dc SQUID," Appl. Phys. Lett., vol. 43, no. 2, pp. 210-212, Jul. 1983.

[6] G. S. Krivoy and H. Koch, "An all thin-film resistive DC SQUID-A current-controlled oscillator," Supercond. Sci. Technol., vol. 5, no. 10, pp. 605-608, Oct. 1992.

[7] G. S. Krivoy, C. Aßmann, M. Peters, and H. Koch, "Design and fabrication of thin-film resistive SQUIDs," J. Low Temp. Phys., vol. 99, no. 1, pp. 107-120, Apr. 1995.

[8] G. S. Krivoy and H. Koch, "Thin-film resistive SQUIDs," IEEE Trans. Appl. Supercond., vol. 5, no. 2, pp. 3244-3247, Jun. 1995.

[9] S. Menkel, C. Aßmann, G. Krivoy, and H. Koch, "An all thin film resistive dc SQUID,” Czech. J. Phys., vol. 46, pp. 2863-2864, 1996.

[10] S. Menkel, D. Drung, C. Aßmann, and T. Schurig, "A resistive d.c. SQUID noise thermometer," Appl. Supercond., vol. 6, no. 7-9, pp. 417-422, 1998.

[11] S. Menkel, D. Drung, Y. S. Greenberg, and T. Schurig, "Integrated thin film dc RSQUIDs for noise thermometry," J. Low Temp. Phys., vol. 120, no. 5, pp. 381-400, Sep. 2000.

[12] S. L. Thomasson and C. M. Gould, "Ultralow temperature SQUIDs for primary thermometry," IEEE Trans. Appl. Supercond., vol. 9, no. 2, pp. 3507-3510, Jun. 1999.

[13] J. C. Macfarlane, L. Hao, D. A. Peden, and J. C. Gallop, "Linewidth of a resistively shunted high-temperature-superconductor Josephson heterodyne oscillator," Appl. Phys. Lett., vol. 76, no. 13, pp. 1752-1754, Mar. 2000.

[14] L. Hao, D. A. Peden, J. C. Gallop, and J. C. Macfarlane, "Tunable HTS rf Josephson-effect oscillator based on YBCO-Au-YBCO resistive SQUID," Supercond. Sci. Technol., vol. 14, no. 12, pp. 1119-1123, Dec. 2001.

[15] J. Macfarlane, J. Du, R. Taylor, and C. Pegrum, "Simulation and measurement of HTS Josephson heterodyne oscillator," IEEE Trans. Appl. Supercond., 2009, To be published.

[16] J. Du, J. C. Macfarlane, and L. Hao, "Noise temperature and linewidthof high-temperature-superconducting heterodyne gigahertz oscillators," Appl. Phys. Lett., vol. 93, no. 3, p. 033507, Jul. 2008.

[17] C. M. Pegrum, "The dynamics of high-frequency DC RSQUID oscillators," Supercond. Sci. Technol., 2009, To be published.

[18] E. S. Fang and T. Van Duzer, "A Josephson integrated circuit simulator (JSIM) for superconductive electronics application," in Extended abstracts of the Int. Superconductivity Electronics Conf. (ISEC'89), Tokyo, Japan, 1989, pp. 407-410.

[19] J. Satchell, "Stochastic simulation of SFQ logic," IEEE Trans. Appl. Supercond., vol. 2, no. 7, pp. 3315-3318, Jun. 1997.

[20] C. M. Pegrum, "Control programs to generate $I-V$ and $V-\Phi$ curves," in Extended abstracts of the Int. Superconductivity. Electronics Conf. (ISEC'97), Berlin, Germany, 1997, pp. 34-36.

[21] S. R. Whiteley, "Josephson-junctions in SPICE3," IEEE Trans. Magn., vol. 27, no. 2, pp. 2902-2905, Mar. 1991.

[22] V. Ambegaokar and B. I. Halperin, "Voltage due to thermal noise in the dc Josephson effect," Phys. Rev. Lett., vol. 22, no. 25, pp. 1364-1366, Jun. 1969.

[23] R. Gupta, Q. Hu, D. Terpstra, G. J. Gerritsma, and H. Rogalla, "Noise study of a high- $T_{c}$ Josephson junction under near-millimeter-wave irradiation," Appl. Phys. Lett., vol. 64, no. 7, pp. 927-929, Feb. 1994.

[24] C. C. Chi, C. Vanneste, W. J. Gallagher, A. W. Kleinsasser, S. I. Raider, and R. L. Sandstrom, "Rf-driven Josephson-junctions: Tails on the Shapiro steps of the $I-V$ curves," Phys. Rev. B, vol. 38, no. 13, pp. 9227-9230, Nov. 1988.

[25] D. C. Cronemeyer, C. C. Chi, A. Davidson, and N. F. Pedersen, "Chaos, noise, and tails on the $I-V$ curve steps of rf-driven Josephson-junctions," Phys. Rev. B, vol. 31, no. 5, pp. 2667-2674, Mar. 1985. 